\title{
A new loading-constraining device for mechanical testing with misalignment auto-compensation
}

\author{
G. Olmi \\ University of Bologna, Engineering Faculty, DIEM Dep., Viale del Risorgimento, 2, 40136 Bologna, Italy \\ giorgio.olmi@unibo.it
}

\begin{abstract}
Experimental testing represents the basic approach for mechanical characterization. Due to the high costs for test execution, the research is often developed with the collaboration of several laboratories, finally collating al the results. At this stage problems may arise due to data scattering, often due to (lateral or angular) misalignments between the fixed and the moving machine crossheads. The object of this work is to manufacture and experimentally validate a novel loading-constraining device for misalignment compensation. Conceptual design is applied to investigate on the optimal technical solution. The developed device achieves misalignment reduction with the use of a thrust bearing with sphered housing washers. The bearing is mounted under one of the specimen heads: the inverted orientation of the upper ring, allowing relative lateral displacement between the two rings, and the spherical shape of the washer compensate for offset and angular misalignments. Experimental results showed that such misalignments are respectively reduced to less than 0.02 $\mathrm{mm}$ and $0.05^{\circ}$, while the bending strain is lowered to just $6 \%$ of the nominal one, so that requirements in different standards for mechanical testing can be fulfilled.
\end{abstract}

KEYWORDS. Mechanical testing; Misalignment compensation; Conceptual design; Thrust bearing; Percentage bending.

\section{INTRODUCTION}

M any types of tests (static, high cycle fatigue, low cycle fatigue, creep, ...) under different loads are nowadays available for material characterization. Due to the high costs, related to machine procurement or maintaining, it is becoming a common practice to develop the research in cooperation with other laboratories, and to share results. At the end of the experimentation the results have to be compared together and collated for the final processing of the whole database. It is at this stage that serious problems may arise due to the scatter of data, mainly due to uncertainties in the load application. Even low cycle fatigue test results, despite their good repeatability for experimentations conducted by the same machine [1-2], can be affected by a great scattering, when the campaign is performed in different laboratories or by using different machines [1-3]. One of the main causes for such uncertainties is surely the misalignment of the test piece with respect to the axial load. The introduction of unexpected bending during axial tests makes results less reliable and accurate, reducing the fatigue strength [4-5]. Possible sources of departing from the ideal condition of a perfectly axial load are a poor alignment in the loading train or a misalignment between the fixed and the moving crossheads of the machine. The first type of misalignment is due to an offset between the centre-lines of the load train with respect to a non rigid machine frame. It may imply a highly damaging bending mechanism, but it can be easily reduced, by increasing the frame stiffness. The second type of misalignment can be related to a lateral or an angular displacement between the machine grips. The Standard [6] recommends carefully checking the misalignment by means of a test specimen, with strain gauges applied along its axis. According to the standard, the maximum bending 
strain should not exceed $5 \%$ of the minimum strain amplitude to be considered in strain controlled conditions fatigue tests. However, no technical solutions are advised for misalignment compensation, apart from the use of Wood's Metal. Other papers, such as [7-8], suggest computing or measuring a percentage bending, i.e. the ratio between the bending and the nominal strain: in some Standards for creep tests a maximum value of $7.5 \%$ is suggested.

However, many experimental campaigns are usually preceded by just a careful mounting, in order to well align the load train [9-12], and sometimes by a brief check to measure the bending component in the unloaded condition only. There are very few papers [11-12], where the problem of misalignment is dealt with, with the suggestion of technical solutions to achieve a compensation of the undesired effects.

The purpose of this research is to develop an original loading-constraining device which is able to compensate for misalignments (lateral or angular) between testing machine grips, so that the requirements in [6] or in [7] for tests under a nominal axial load can be met.

\section{CONCEPTUAL DESIGN AND TECHNICAL FEATURES}

he approach of conceptual design [13] was initially applied in the analysis of different devices for specimen clamping and misalignment compensation and in the following comparative analysis for the choice of the fittest one. In the first stage a QFD (Quality Function Deployment) was run for listing the major design requirements which are the safety in load transmission, the precision at misalignment compensation and device versatility, i.e. its ability to be mounted on testing machines of different models. Another important aspect was detectability: it was decided to perform a full check on device effectiveness at misalignment compensation by the experimental measurement of bending and misalignment offsets.
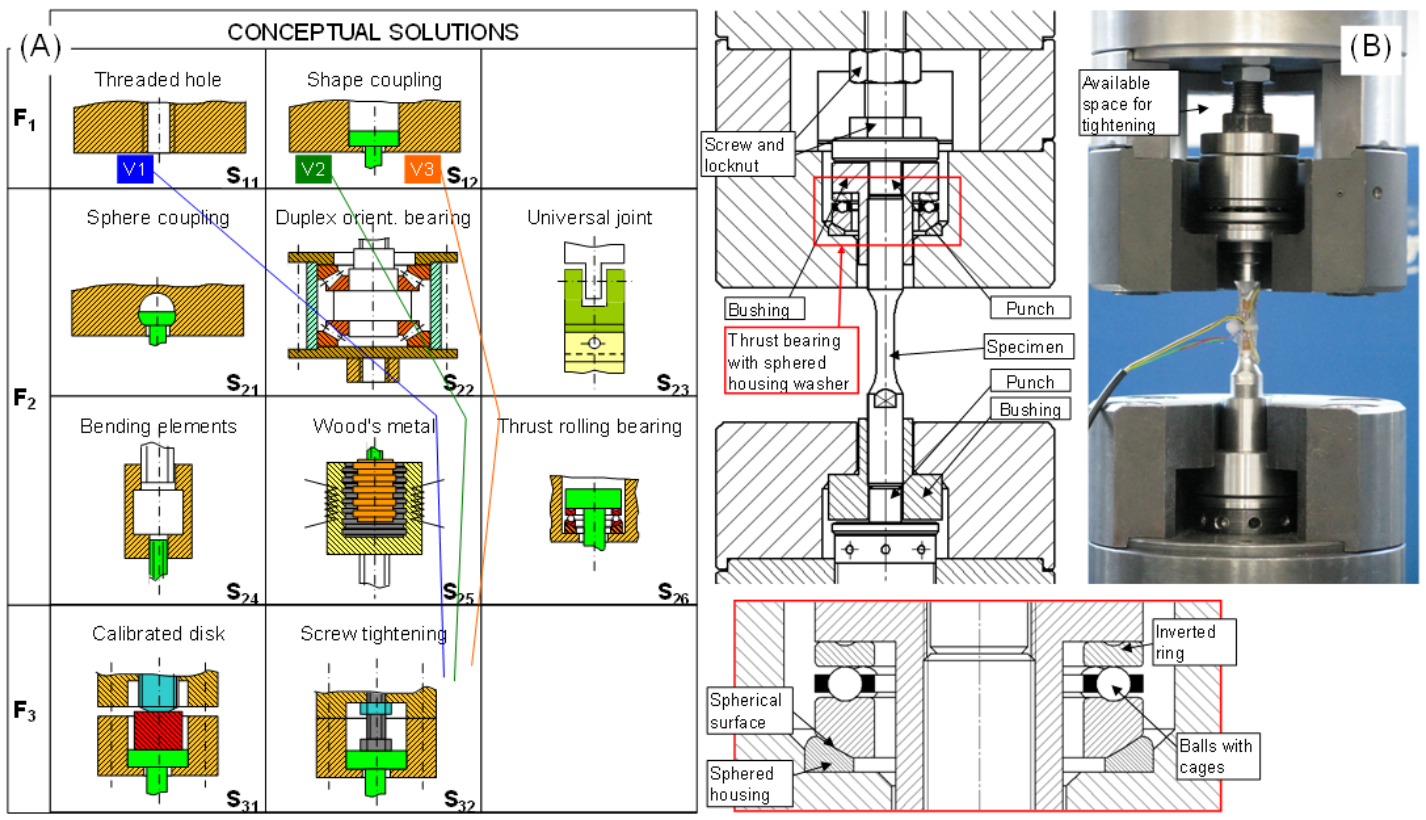

Figure 1: Morphological matrix of the conceptual solutions (A) and a photo and some drawings of the proposed device (B).

The following step consisted in functional analysis, i.e. in the research of the main device functions with the simultaneous determination of conceptual solutions, i.e. of design options, able to fulfil the aforementioned functions. Three main functions, referred to as $F_{1}, F_{2}$ and $F_{3}$, were detected. The first one, $F_{1}$, refers to full constraining at one specimen end, while the second one, $\mathrm{F}_{2}$, regards constraining with the possibility of adjustment (to prevent or reduce misalignment) at the other end. Finally, function $\mathrm{F}_{3}$ involves a slight pre-compression at the specimen ends to prevent any clearance run at load inversion. The matrix in Fig. 1A shows the conceptual solutions that were considered for the comparative analysis. It can be observed that conceptual solutions for function $F_{1}$ account for different specimen geometries, with threaded or button-head connections at the ends [6]. Function $F_{2}$ is surely much more difficult to achieve: the first option to be considered was to make use of a spherical coupling [14], however, it requires a careful lubrication, moreover is not suitable 
for compressive load transmission. The following solution is similar to the previous one, but in this case the hemispherical coupling is achieved by using two spherical roller thrust bearings posed at mirrored locations. They can be adequately preloaded, to prevent any clearance run at load inversion, however any minimal imperfection during mounting could imply misalignments between the bearing centres and the specimen axis. The universal joints [15-16] (double cardanic joints) are not suitable for compressive load transmission. In [17] the use of members with a high bending compliance is advised, but they have the drawback that instability effects affecting the loading train may occur during testing under compression. A further option, the most recommended in [6] is to make use of Wood's Eutectic metal, to freeze the specimen in the ideally perfectly aligned position. However, serious drawbacks are present: Wood's metal has toxic properties and a quite low shear strength, which makes it unsuitable for high load transmission, especially in low cycle fatigue tests. Finally, the last solution suggests making use of a thrust ball bearing, where one of the rings must be mounted in inverted position beneath the specimen head. In addition, with the use of a thrust bearing with sphered housing washers, not only transverse offset but also angular misalignments between machine clamps can be compensated. Coming to the last function $\mathrm{F}_{3}$, this can be achieved by impressing a pre-compression state on the specimen heads. For this purpose, different solutions are available, involving calibrated disks or wedges or a cylindrical member (threaded screw) to be lowered towards the specimen upper end, until contact and pre-load are achieved.

On the basis of the matrix containing the conceptual solutions for functions $F_{1}, F_{2}, F_{3}$ (Fig. 1A), three variants (V1, V2, V3) were considered for the comparative analysis of value. This analysis led to the choice of option V3, whose final manufacturing is shown in Fig. 1B. The specimen lower end is completely fixed: the threaded head is connected to a bushing, having an external diameter coupled to the machine clamp slot with a strict tolerance. Pre-compression of the horizontal specimen surface towards a punch and a cylindrical element of the machine clamp is performed by means of a wrench. The specimen is then constrained at its upper side: as for the lower side, a bushing is connected to the threaded head. The bushing is placed on the inverted ring of a thrust bearing with sphered housing washers. The upper head precompression is finally achieved, by lowering a screw, having a machined head, and by then fixing it in its final position by a locknut. The screw transmits an adequate pre-load to a punch, acting on the upper head. The described device is able to work with or without lubrication, it is however advisable to place a thin lubricant layer at the interface between the hemispherical surface and the sphered housing. A member, connected to an existing clamp, was designed so that an internal space could be made available for the tightening procedure by using a wrench.

\title{
EXPERIMENTAL PROCEDURE AND RESULTS
}

\begin{abstract}
A possible criticality of the developed solution is that it is not able to completely cancel all the lateral and angular misalignments: for this reason an experimental campaign was planned for a direct estimation of these offsets, and in order to compare the bending contribution to the nominal axial load. A specimen, made in ASTM A470, manufactured according to [6], was equipped with four electrical strain gauges, applied along the longitudinal direction. They were two-by-two connected to form two Wheatstone half bridges, for measurement of the bending strain state, with natural cancellations of the contributions due to the axial loads. The tests were conducted on an INSTRON 8032 machine with static application of tensile and compressive loads in load controlled conditions. The considered range varied from $25 \mathrm{kN}$ (compression) to $+25 \mathrm{kN}$ (tension) with a variation step of $1 \mathrm{kN}$. At each load step, the voltage outputs of the two Wheatstone bridges were recorded by a suitable acquisition device (P3 Strain Indicator, Vishay, Wendell, NC, USA). The structure composed of the specimen and its constraints (it is completely fixed at its lower end and constrained with the adjustment option due to the thrust bearing with sphered housing washers at its upper side) acts as a cantilever beam. The specimen with strain gauge instrumentation acts as a two-degree of freedom loading cell, able to measure the shear force and the bending moment on the specimen itself. By the knowledge of these two actions, it is then possible to compute the shear displacement and the rotation at the free edge, respectively corresponding to the lateral offset and to the angular misalignment. The experimentation was conducted by mounting the instrumented specimen, according to the aforementioned procedure. The static loading procedure, firstly in tension and then in compression, was repeated for three times for statistical evidence reasons. Afterwards, the specimen was dismounted, rotated by $90^{\circ}$ and re-mounted to investigate the mechanical actions and misalignments in the perpendicular plane, by repeating the same procedure. Again, the same methodology was used to repeat the experimentation in the no-bearing condition, in order to investigate the efficiency of the proposed bearing device at reducing misalignments and specimen bending. All the results, related to total lateral $(\eta)$ and angular $(\varphi)$ misalignments and percentage bending (bending strain $\left(\varepsilon_{\mathrm{b}}\right) /$ nominal strain due to the axial load $\left(\varepsilon_{a}\right)$ ) are shown in Fig. 2. Due to the great repeatability and linearity of the strain gage readings, when using the proposed device, related results were interpolated and extrapolated to the $-40 \div+40 \mathrm{kN}$ force range.
\end{abstract}




\section{DISCUSSION AND FINAL REMARKS}

he approach of conceptual design was here used to develop a novel loading-constraining device, having the basic feature of using a thrust bearing with sphered housing washers with an inverted ring. It is located beneath the specimen upper head, making it possible for the specimen to get auto-aligned, with the consequent compensation of both lateral and angular misalignments between the machine clamps. The efficiency at misalignment reduction was experimentally investigated by using an instrumented specimen [6], trying to reproduce the actual testing conditions. The most remarkable results, together with a comparison to those in the no-bearing configuration, can be so summarized:

$\checkmark$ The lateral misalignment is about $0.01-0.02 \mathrm{~mm}$, respectively with a $44 \%$ and a $30 \%$ reduction, for tensile and compressive loads, with respect to the no-bearing configuration.

$\checkmark$ The angular misalignment is lower than $0.05^{\circ}$, with a $28 \%$ reduction for tensile loads and a $6 \%$ reduction for compressive ones.

The percentage bending tends to decrease, as the applied force increases, to values around $6 \%$ (with a reduction by $58 \%$ and $50 \%$ for tensile and compressive loads): this value appears to be acceptable also on the basis of the remarks contained in $[6-7]$.

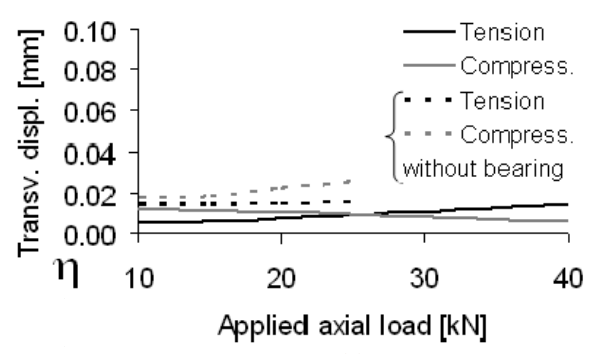

(a)

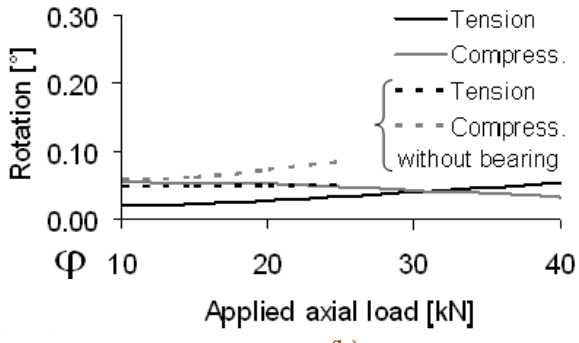

(b)

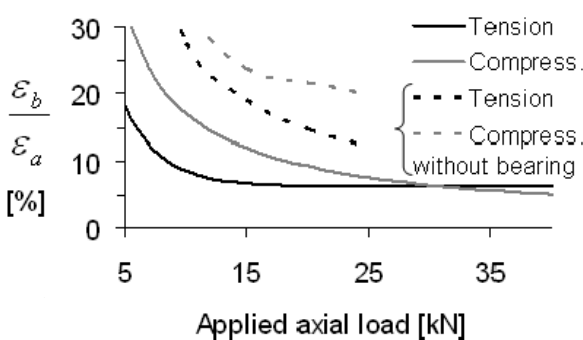

(c)

Figure 2: Lateral (a) and angular (b) misalignments and percentage bending (c).

\section{REFERENCES}

[1] F.A. Kandil, B.F. Dyson, Fatigue Fract. Engng. Mater. Struct., 16 (5) (1993) 509.

[2] F.A. Kandil, B.F. Dyson, Fatigue Fract. Engng. Mater. Struct., 16 (5) (1993) 529.

[3] G. B. Thomas, R. K. Varma, Harmonisation of Testing Practice For High Temperature Materials (Edited by M.S. Loveday, T.B. Gibbons), Elsevier Science Publishers, (1992) 155.

[4] M. L. Roessle, A. Fatemi, International Journal of Fatigue, 22 (2000) 495.

[5] P. Hähner et al., International Journal of Fatigue, 30 (2008) 372.

[6] ASTM E606-04, Standard Practice for Strain-Controlled Fatigue Testing (2004).

[7] A. K. Schmieder, A. T. Henry, Elevated Temperature Testing Problem Areas, STP 488 (1971) 43.

[8] J. Schijve, G. Campoli, A. Monaco, International Journal of Fatigue, 31 (2009) 1111.

[9] M. L. Roessle, A. Fatemi, International Journal of Fatigue, 22 (2000) 495.

[10] R. Cortez, S. Mall, J. R. Calcaterra, International Journal of Fatigue, 21 (1999) 709.

[11] A. Fatemi, Z. Zeng, A. Plaseied, International Journal of Fatigue, 26 (2004) 663.

[12] T. R. Vantiger, R. I. Stephens, M. Karadag, International Journal of Fatigue, 24 (2002) 1275.

[13] G. Pahl, W. Beitz, Engineering Design, a Systematic Approach, Springer-Verlag, Berlin, Germany (1997).

[14] B. W. Christ, Metallurgical Transactions, 4 (8) (1973) 1961.

[15] R. K. Penny, E. G. Ellison, G. A. Webster, Material Research and Standards, 6 (2) (1966) 76.

[16] R. J. O’Kane, American Society for Testing and Materials, STP 488, (1971) 61.

[17] G. Birkbeck, N. J. Petch, D. M. Rae, Journal of Iron and Steel Institute, (1972) 675. 